\title{
A Case Report on Metastatic Thyroid Carcinoma: Radiation-induced Bystander or Abscopal Effect?
}

Slavisa Tubin*, Franco Casamassima, Claudia Menichelli, Gabriella Pastore, Alessandro Fanelli and Rossana Crisci

Istituto Ricerche Cliniche Ecomedica, Empoli, Florence, Italy

\begin{abstract}
For the first time the rare radiobiological phenomenon of spontaneous regression of an unirradiated tumor in a course of irradiation of other, distant lesion in a patient affected by thyroid carcinoma has been observed. Identifying tumors that are capable of inducing this phenomenon, and doses for which its intensity would be maximum, this effect could be exploited as a powerful weapon for radio-sensitize the regional or distant tumor and increase the control of it.
\end{abstract}

Keywords: Radiation induced abscopal effect; Radiation induced bystander effect; Thyroid cancer

\section{Introduction}

The effects of radiation on human tissues are not just local as once believed but they can appear even outside of the irradiated field [1-5]. Based on what has been published, both from in vitro and in vivo studies $[6,7]$, it is known that those effects are at least regional. The irradiated tumor tissue may communicate, in some way, radiation effects to the not irradiated neighboring or distant tumor. If the irradiated tumor is forming a physical contact with a not irradiated tumor, then the phenomenon of transmission of the radiation damage is called "bystander effect". The "abscopal effect" [8], on the other hand, refers to the phenomenon of damage communication between irradiated tumor and a distant tumor that has not been irradiated. The first report on abscopal effect dating from 1953 was published by Mole [1]. Thereafter many authors have published observations on in vivo abscopal effects in different tumor types, such as malignant melanoma [2], lymphoma [3], hepatocellular carcinoma [4], and cervical carcinoma [5]. So far no authors have published on such phenomena induced by thyroid carcinoma. This is the first case report on a radiation-induced abscopal effect in a thyroid cancer-patient.

\section{Methods and Materials}

The patient, a 72 year old Italian man, with negative family history for cancer, was diagnosed in 2007 with medullary thyroid carcinoma. He was treated at the "Endocrinology and Kidney-Integrated Activity Department" in Pisa, Italy. He is an ex smoker who stopped smoking in 1983, with a history of rheumatic aortic and mitral valve stenosis (with mechanical prosthesis implants), chronic atrial fibrillation (with a permanent pacemaker implant), benign prostatic hypertrophy, gastroesophageal reflux, diverticular disease of sigma, arterial hypertension and chronic obstructive bronchopneumopathie. None has been previously subjected to radiation treatment.

In 2007 he underwent left supraclavicular lymph node biopsy due to swelling in that region. Pathological examination revealed a medullary thyroid carcinoma. He underwent total thyroidectomy with left neck, pretracheal and superior mediastinal lymph node dissection in January of 2008. Subsequent pathological examination confirmed multifocal, bilateral medullary thyroid carcinoma with maximum diameter of $2 \mathrm{~cm}$, stage pT3 pN1b IVA; 16 of 31 examined lymph nodes were positive for metastases. Clinical staging at that time did not show any other lymph node or organ involvement.
Nine months later, in September of 2008, he underwent left infraclavicular lymph node dissection for the appearance of metastases in that region. In 2011, he was treated with an experimental drug inhibitor of tyrosine-kinase receptor (known as XL 184), for new disease progression at mediastinal lymph node levels $4 \mathrm{R}$ and 6 . That treatment was carried out from February until March, when it was discontinued for the occurrence of important side effects.

He remained under close medical supervision. A CT-scan performed in March of 2012 showed further mediastinal lymph nodes enlargement at levels $4 \mathrm{R}$ and 6 that measured $29 \times 28 \mathrm{~mm}$ and $66 \times 48$ $\mathrm{mm}$, respectively. No other site involvement was found at that time.

The patient was given the option to again enter the experimental study with the inhibitor of tyrosine-kinase receptor, but considering the side effects during previous treatment he chose not to participate. Instead the patient was monitored closely, allowing the possibility of further treatment in case of disease progression. In June of 2012 he was hospitalized for worsening dyspnea, caused by obstruction of the bronchi by the mediastinal mass. At that time the CT scan showed the already known mediastinal lymphadenopathies to be larger than in the previous scans. Maximum diameter for the lymph node station level $4 \mathrm{R}$ was $40 \mathrm{~mm}$ vs. $29 \mathrm{~mm}$ and for the station level 6 was $80 \mathrm{~mm}$ vs. 66 $\mathrm{mm}$. Due to his worsening condition, he was sent to our department for radiotherapy.

Examination of the patient revealed that his general condition was fair with Karnofsky performance status 90 . He complained of moderate dyspnea and no other disorders. We decided to give him symptomatic radiotherapy treatment for the purpose of bronchial decompression. We defined the two symptomatic lymphadenopathies at the lymph node station level $4 \mathrm{R}$ and 6 (Figure 1) as the target for irradiation. Given that medullary thyroid carcinoma is a radioresistant disease, we

*Corresponding author: Slavisa Tubin, Istituto Ricerche Cliniche Ecomedica, Via Cherubini n.c. 2, 50053 Empoli, Florence, Italy, Tel: 0039-0571-992-765/0039-328 776-9106; Fax: 0039-0571-992-726; E-mail: s.tubin@radioterapia.ecomedica.it

Received October 25, 2012; Accepted November 21, 2012; Published November 23, 2012

Citation: Tubin S, Casamassima F, Menichelli C, Pastore G, Fanelli A, et al. (2012) A Case Report on Metastatic Thyroid Carcinoma: Radiation-induced Bystander or Abscopal Effect? J Cancer Sci Ther 4: 408-411. doi:10.4172/1948-5956.1000175

Copyright: ( 2012 Tubin S, et al. This is an open-access article distributed under the terms of the Creative Commons Attribution License, which permits unrestricted use, distribution, and reproduction in any medium, provided the original author and source are credited. 


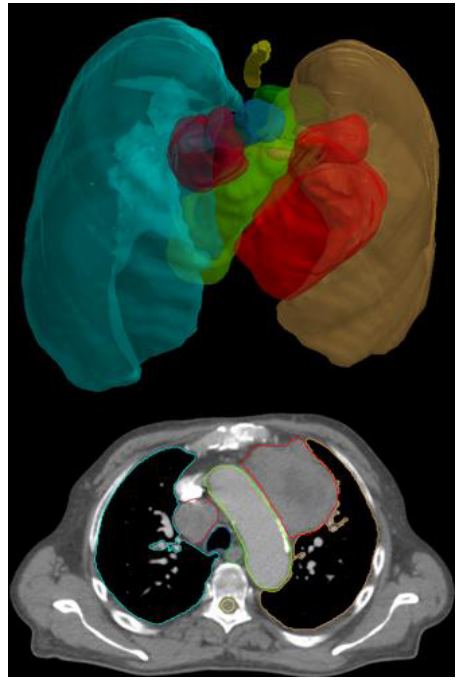

Figure 1: CT simulation scan shows two mediastinal lymphadenopathies: the smaller at level 4R (inductor of bystander effect) was irradiated with 3000 $\mathrm{cGy}$, and the larger one at level 6 (bystander effect responder) remained unirradiated [20].

prescribed a total dose of $3000 \mathrm{cGy}$ in 3 fractions to the $70 \%$ isodose line at the $4 \mathrm{R}$ lymph node level (the smaller lymphadenopathy group). We used the Volumetric modulated arc therapy (VMAT) technique and 6 megavolt energy photons. The Gross tumor volume (GTV) was contoured with an additional margin of $4 \mathrm{~mm}$. We started the treatment on June $25^{\text {th }}$ by irradiating the smaller volume at level $4 \mathrm{R}$ first, because of more evident bronchial compression on that site. The lymphadenopathy at level 6 was not irradiated at the time, and was located outside of the radiation field. Only a very low percentage of that lesion, precisely $5.97 \%$, received 500 cGy in 3 fractions (Figure 2), and the received mean dose was 264 cGy.

\section{Results}

One month after that treatment, our patient returned to treat the other lymphadenopathy at level 6. A cone beam CT was performed before treatment, showing a size reduction of the treated lymphadenopathy $4 \mathrm{R}$, indicating a partial response at the time. Surprisingly, the lymphadenopathy at level 6 had also reduced in size and to a greater degree than the treated $4 \mathrm{R}$-lesion, thereby showing a response to "no treatment" (Figure 3). This untreated lesion had shrunk in anteroposterior (AP) and lateral (LL) directions with a maximum size reduction of $1.6 \mathrm{~cm}$ and a $32 \%$ volume reduction $(171,5 \mathrm{cc}$ vs. 117 cc). A simulation CT scan was repeated to re-plan the treatment for this lesion, which was then irradiated from August $14^{\text {th }}$ to August $20^{\text {th }}$ of 2012, with 4000 cGy in 5 fractions prescribed at 70\% isodose line.

\section{Discussion}

Although, there are many published cases on radiation-induced abscopal effect, there has been no description in literature of such a phenomenon in a patient with primary thyroid cancer. It is unclear whether what we observed was an abscopal or bystander effect. What we do know is that we observed a partial response at the level of nodal mass irradiated unintentionally (with a very low dose that covered a very small percentage of its volume) as a result of irradiation of another distant metastatic lymph node lesion, without physical contact between them. There are no other plausible reasons which could trigger such a reaction because our patient neither received chemotherapy, nor drugs different from those with which he was being treated before the course of radiotherapy. As previously stated, the bystander effect denotes the transmission of radiation damage from irradiated tumor to adjacent non-irradiated tumor through inter-cellular gap junctions. Another mechanism of radiation damage "communication" from the irradiated tumor to the non-irradiated tumor occurs via "bystander factors". In this model, soluble cytokine-like molecules would function as messengers between distant tumors, without a need for physical contact between them. While there are several techniques to demonstrate the mechanism of inter-tumor communication in response to irradiation in vitro [7], it is much more complex to demonstrate the responsible mechanisms in vivo due to the complexity of a system such as the human organism. The mechanisms of these phenomena have not been clearly understood. There are two mechanisms proposed by Lin et al. [9], the ischemia and immune response. Stamell et al. [10] have found an association between the abscopal effect and the systemic antitumor immune response in melanoma. Irradiating only the primary melanoma resulted in the complete response in all in-transit metastases and was associated with anti-MAGE-3 antimelanoma antibodies that were found upon serological testing. Other hypotheses have also been proposed. Hall et al. [11] suggested that in response to local irradiation there is an increase in the level of serum Tumor Necrosis Factor alpha,
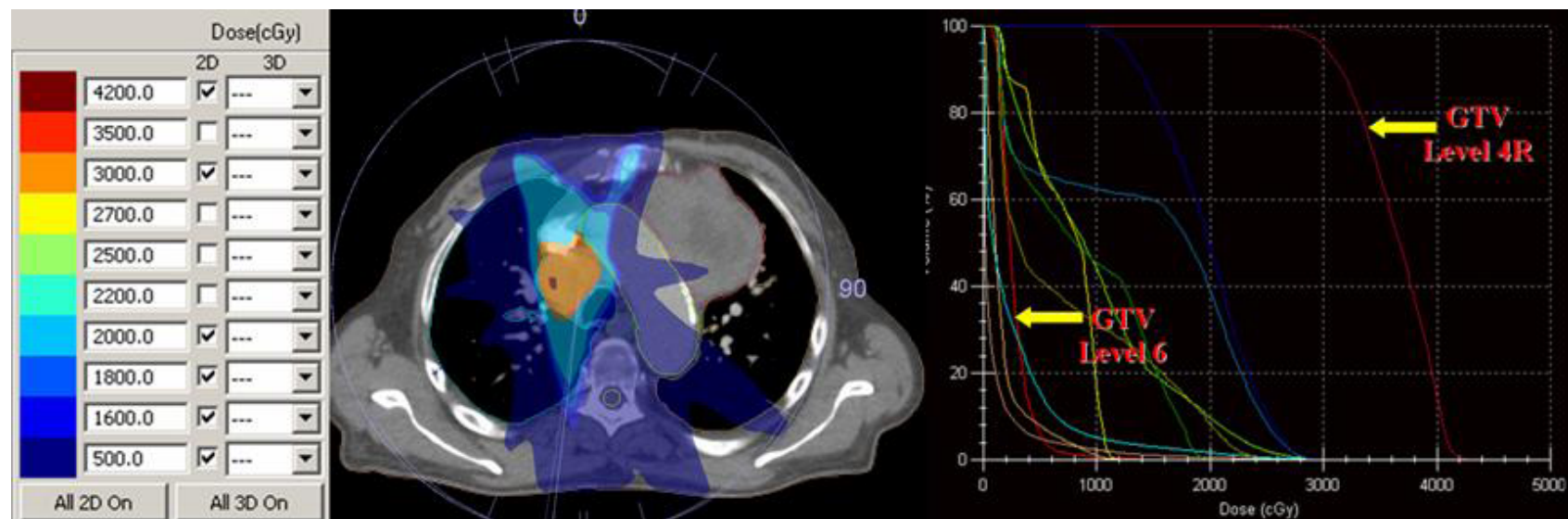

Figure 2: Dose distribution and DVH of treatment plan for irradiation of lymph node level $4 \mathrm{R}$ show the minimum dose that covers the small volume of untreated lymphadenopathy at level 6. 
Citation: Tubin S, Casamassima F, Menichelli C, Pastore G, Fanelli A, et al. (2012) A Case Report on Metastatic Thyroid Carcinoma: Radiationinduced Bystander or Abscopal Effect? J Cancer Sci Ther 4: 408-411. doi:10.4172/1948-5956.1000175
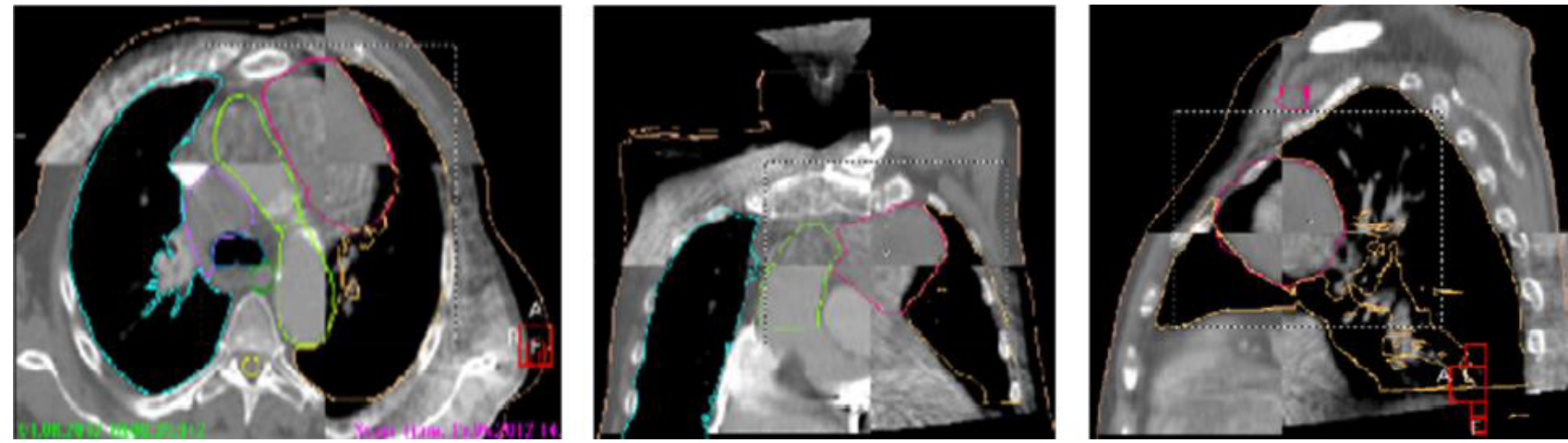

Figure 3: Cone beam CT shows size reduction in untreated lesion at level 6 one month after irradiation of lymphadenopathy at level 4R.

which in turn mediates the antimitotic systemic effect even at sites distant from the irradiated tumor. The local irradiation could destroy tumor tissue and cause the release of tumor antigens into the systemic circulation. These antigens would be capable of stimulating the natural killer-mediated immune response, against a tumor, distant from the irradiation site that expresses the same antigens [12]. As we did not expect such an effect, we did not create a preemptive setup to measure the mechanisms involved.

We analyzed a lymphadenopathy size reduction in AP, LL and craniocaudal diameters, trying to understand if there was a correlation between a size reduction direction and dose distribution that covered "not irradiated" tumor. We expected that if the mechanism responsible for the response of the tumor was inter-cellular gap junction-mediated communication (bystander effect), then the gradient of the size reduction of the tumor should be more asymmetric or concentrated on the same side where that minimum dose reached the tumor (Figure 2). We did not find any such correlation. Maximum size reduction was found to be $1.6 \mathrm{~cm}$ in both directions, AP and LL; the CC tumor extension did not change. The tumor had shrunk in a uniform manner (Figure 4). The volume of lymphadenopathy measured before the radiotherapy treatment was $171.5 \mathrm{cc}$ vs. $117 \mathrm{cc}$ one month after irradiation of $4 \mathrm{R}$ lymph node level, which equals a $32 \%$ volume reduction. It is not only the size reduction that we observed, but also the increase in diameter of central tumor necrosis (Figure 4). It seems that a factor that reacted in response to radiation in the $4 \mathrm{R}$, interacted with the non-irradiated tumor uniformly, indicating a mechanism more likely to be an abscopal effect. From another point of view, as the dose applied to this cancer was very low and covered just a small portion of its mass, it is unlikely that this could cause a bystander effect so powerful to reduce the volume of mass by $32 \%$. Only $5.97 \%$ of tumor received $500 \mathrm{cGy}$ in 3 fractions. The bystander effect is mainly observed in in vitro experiments on cell cultures as induction of sisterchromatid exchanges, mutations, neoplastic transformation, apoptosis, micronucleus, chromosomal aberrations and DNA double strand breaks, using very low doses of alpha particles (range: mGy, cGy) [13], but also after conventional irradiation (X-rays, gamma rays) at low as well as at conventional doses [14]. Of the limited number of in vivo accounts, some report that there was an observed bystander effect with dramatic reduction in tumor size, for a small number of patients whose large masses were irradiated partially in one fraction with high doses using GRID radiotherapy [15,16]. Peter et al. [17] reported on a robust abscopal effect caused by this technique in distant tumors that were not irradiated. The tumor in our case study was irradiated partially, in only two very small islands, each of which received 3 fractions of
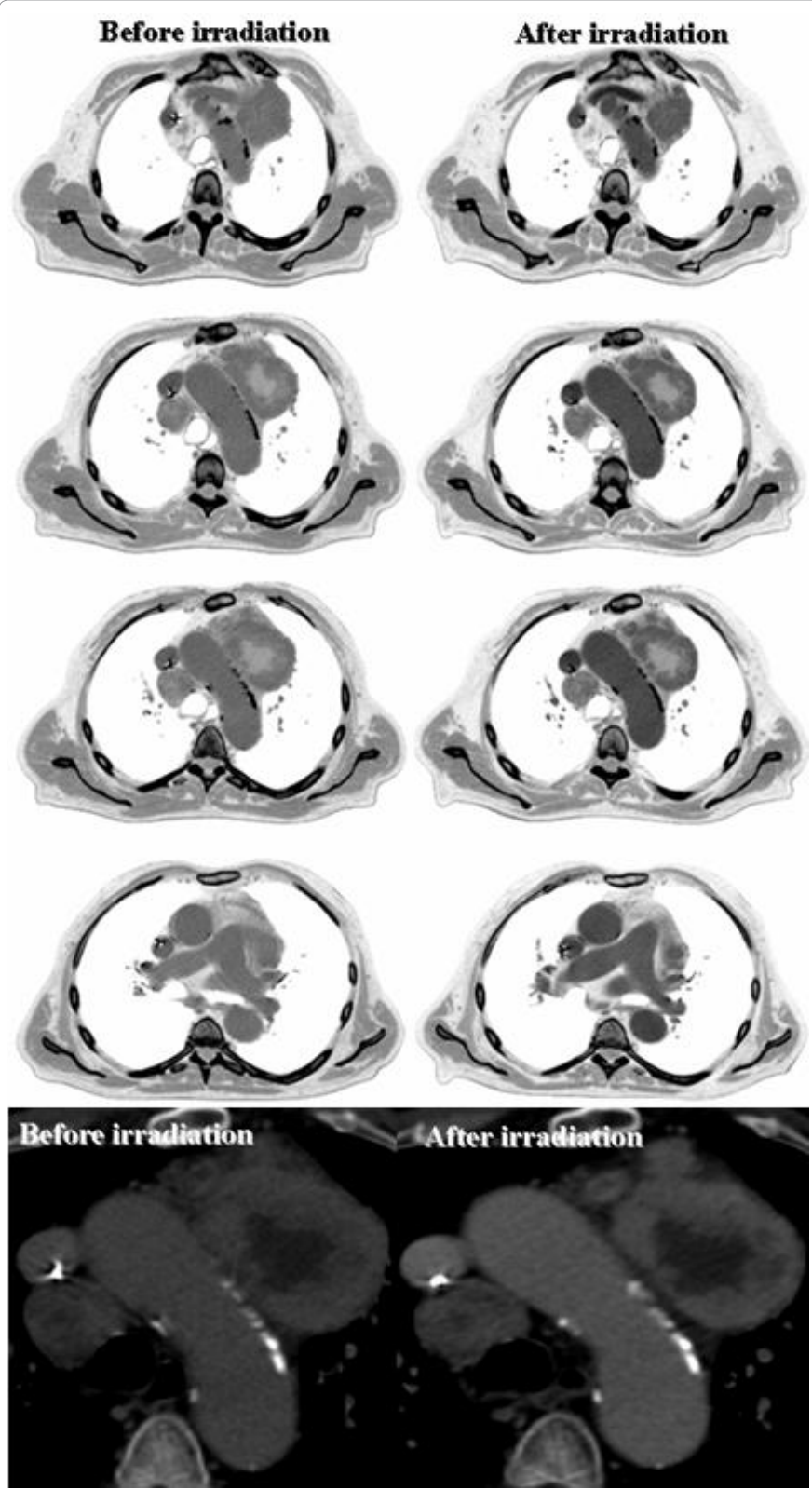

Figure 4: Size reduction and increase of central tumor necrosis of lymphadenopathy at level 6 before and after irradiation. 
Citation: Tubin S, Casamassima F, Menichelli C, Pastore G, Fanelli A, et al. (2012) A Case Report on Metastatic Thyroid Carcinoma: Radiationinduced Bystander or Abscopal Effect? J Cancer Sci Ther 4: 408-411. doi:10.4172/1948-5956.1000175

167 cGy. That dose is much lower than that used in published studies on GRID radiotherapy; the irradiated volume (islands) was also much smaller. That would not be enough to cause a partial response for a tumoral mass with this volume. It would not fit with the current model and published data on linear cell killing. Bystander factors such as TNF-alpha [18,19], TRAIL [19] and Ceramide [20] are induced in cells that are under the open field of the high-dose GRID areas and are hypothesized to be responsible for initiating the cell death cascade both in the epithelial and endothelial compartments of the tumor microenvironment. It is reasonable to assume in our case that both effects were involved: bystander and abscopal.

\section{Conclusion}

We observed a rare radiobiological phenomenon of tumor shrinkage in a site distant to the area of irradiation, in a type of tumor previously not associated with these phenomena (medullary thyroid carcinoma). This tumor responded to signals induced by irradiation of the other distant tumor likely due to mixed bystander and abscopal effects. With a better understanding of factors responsible for the transmission of radiation-induced damage and which doses would be able to induce such an effect, we would have better guidelines for creating bystander and abscopal effects, to enhance control of microand macroscopic regional and distant disease.

\section{References}

1. Mole RH (1953) Whole body irradiation; radiobiology or medicine? Br J Radiol 26: $234-241$

2. Kingsley DP (1975) An interesting case of possible abscopal effect in malignant melanoma. Br J Radiol 48: 863-866.

3. Rees GJ (1981) Abscopal regression in lymphoma: a mechanism in common with total body irradiation? Clin Radiol 32: 475-480.

4. Oquiñena S, Iñarrairaegui M, Vila JJ, Alegre F, Zozaya JM, et al. (2009) Spontaneous regression of hepatocellular carcinoma: three case reports and a categorized review of the literature. Dig Dis Sci 54: 1147-1153.

5. Takaya M, Niibe Y, Tsunoda S, Jobo T, Imai M, et al. (2007) Abscopal effect of radiation on toruliform para-aortic lymph node metastases of advanced uterine cervical carcinoma--a case report. Anticancer Res 27: 499-503.

6. Chai Y, Hei TK (2008) Radiation Induced Bystander Effect in vivo. Acta Med Nagasaki 53: S65-S69.

7. Azzam El, Little JB (2004) The radiation-induced bystander effect: evidence and significance. Hum Exp Toxicol 23: 61-65.

8. Goldberg Z, Lehnert BE (2002) Radiation-induced effects in unirradiated cells: a review and implications in cancer. Int J Oncol 21: 337-349.

9. Lin TJ, Liao LY, Lin CL, Shih LS, Chang TA, et al. (2004) Spontaneous regression of hepatocellular carcinoma: a case report and literature review. Hepatogastroenterology 51: 579-582.

10. Stamell EF, Wolchok JD, Gnjatic S, Lee NY, Brownell I (2012) The abscopal effect associated with a systemic anti-melanoma immune response. Int $\mathrm{J}$ Radiat Oncol Biol Phys.

11. Hall E (2000) Radiobiology for the Radiologist. Philadelphia: Lippincott, Williams and Wilkins 5: 588 .

12. Demaria S, Ng B, Devitt ML, Babb JS, Kawashima N, et al. (2004) lonizing radiation inhibition of distant untreated tumors (abscopal effect) is immune mediated. Int J Radiat Oncol Biol Phys 58: 862-870.

13. Azzam El, de Toledo SM, Gooding T, Little JB (1998) Intercellular communication is involved in the bystander regulation of gene expression in human cells exposed to very low fluences of alpha particles. Radiat.Res 150: 497-504.

14. Azzam El, de Toledo SM, Little JB (2003) Oxidative metabolism, gap junctions and the ionizing radiation-induced bystander effect. Oncogene 22: 7050-7057.
15. Mohiuddin M, Fujita M, Regine WF, Megooni AS, Ibbott GS, et al. (1999) High-dose spatially-fractionated radiation (GRID): a new paradigm in the management of advanced cancers. Int J Radiat Oncol Biol Phys 45:721-727.

16. Zwicker RD, Meigooni A, Mohiuddin M (2004) Therapeutic advantage of grid irradiation for large single fractions. Int J Radiat Oncol Biol Phys 58: 1309-1315.

17. Peters ME, Shareef MM, Gupta S, Zagurovskaya-Sultanov M, Kadhim M, et al. (2007) Potential utilization of bystander/abscopal-mediated signal transduction events in the treatment of solid tumors. Curr Signal Transd Ther 2: 129-143.

18. Sathishkumar S, Dey S, Meigooni AS, Regine WF, Kudrimoti MS, et al. (2002) The impact of TNF-alpha induction on therapeutic efficacy following high dose spatially fractionated (GRID) radiation. Technol Cancer Res Treat 1: 141-147.

19. Shareef MM, Cui N, Burikhanov R, Gupta S, Satishkumar S, et al. (2007) Role of tumor necrosis factor-alpha and TRAIL in high-dose radiation-induced bystander signaling in lung adenocarcinoma. Cancer Res 67: 11811-11820.

20. Sathishkumar S, Boyanovsky B, Karakashian AA, Rozenova K, Giltiay NV et al. (2005) Elevated sphingomyelinase activity and ceramide concentration in serum of patients undergoing high dose spatially fractionated radiation treatment: implications for endothelial apoptosis. Cancer Biol Ther 4: 979-986. 